\title{
OPTIMAL CONSUMPTION AND INVESTMENT PROBLEM WITH REGIME-SWITCHING AND CARA UTILITY
}

\author{
YONG HYUn SHIN*
}

\begin{abstract}
We use the dynamic programming method to investigate the optimal consumption and investment problem with regimeswitching. We derive the optimal solutions in closed-form with constant absolute risk aversion (CARA) utility.
\end{abstract}

\section{Introduction}

After the pioneer work of Merton [3, 4], portfolio optimization problem has been one of the most important and active area in mathematical finance. Recently, portfolio selection problems combined with regimeswitching are widely considered. $([1,6,5])$

In this paper we investigate the optimal consumption and investment problem with regime-switching under the dynamic programming framework of Karatzas et al. [2]. We use the constant absolute risk aversion (CARA) utility function to obtain the optimal solutions in closed-form.

\section{The financial market}

Let us define a standard Brownian motion $B_{t}$ and a continuous-time two-state Markov chain $\epsilon_{t}$ on a probability space $(\Omega, \mathcal{F}, \mathbb{P})$. We assume that $B_{t}$ and $\epsilon_{t}$ are independent and the filtration $\left\{\mathcal{F}_{t}\right\}_{t \geq 0}$ is generated by both the Brownian motion $B_{t}$ and the Markov chain $\epsilon_{t}$.

We assume that two assets are traded in the financial market: One is a riskless asset with constant interest rate $r$ and the other is a risky asset. We assume that there are two regimes 1,2 in the market and regime $i$ switches into regime $j$ at the first jump time of an independent

Received September 06, 2012; Accepted November 09, 2012.

2010 Mathematics Subject Classification: Primary 91G10.

Key words and phrases: regime-switching, CARA utility, portfolio optimization. 
Poisson process with intensity $\lambda_{i}$, for $i, j \in\{1,2\}$. Here two states of regimes 1,2 are economically considered as bull market and bear market. So, in regime $i \in\{1,2\}$, the risky asset price process follows $d S_{t} / S_{t}=\mu_{i} d t+\sigma_{i} d B_{t}$. The market price of risk is defined by $\theta_{i} \triangleq$ $\left(\mu_{i}-r\right) / \sigma_{i}, i=1,2$. Let $\pi_{t}$ be the $\mathcal{F}_{t}$-progressively measurable portfolio process, the dollar-amount of the agent's wealth invested in the risky asset at time $t$ and $c_{t}$ be the nonnegative $\mathcal{F}_{t}$-progressively measurable consumption process at time $t$. We assume that they hold the following technical conditions:

$\int_{0}^{t} c_{s} d s<\infty$ and $\int_{0}^{t} \pi_{s}^{2} d s<\infty$, for all $t \geq 0$, almost surely (a.s.).

The agent's wealth process $X_{t}$ at time $t$ follows

$$
d X_{t}=\left[r X_{t}+\pi_{t}\left(\mu_{i}-r\right)-c_{t}\right] d t+\sigma_{i} \pi_{t} d B_{t}, \quad X_{0}=x>0, i=1,2 .
$$

\section{The optimization problem}

The agent's optimization problem with CARA utility $u(c):=-e^{-\gamma c} / \gamma$, is to maximize her expected utility

$$
V_{i}(x)=\sup _{(c, \pi) \in \mathcal{A}(x)} \mathbb{E}\left[-\int_{0}^{\tau_{i}} \frac{e^{-\rho t-\gamma c_{t}}}{\gamma} d t+e^{-\rho \tau_{i}} V_{j}\left(X_{\tau_{i}}\right)\right],
$$

where $\tau_{i}$ is the first jump time since the beginning of regime $i, \rho>0$ is a subjective discount rate, $\gamma>0$ is absolute risk aversion and $\mathcal{A}(x)$ is an admissible set of pairs $(c, \pi)$ at $x$, where $i, j \in\{1,2\}$ and $i \neq j$.

Assumption 3.1.

$$
\rho-r+\frac{\theta_{i}^{2}}{2}>0, i \in\{1,2\} .
$$

Next theorem gives our main results.

Theorem 3.2. The value function is given by

$$
V_{i}(x)=-\frac{M_{i}}{\gamma r} e^{-\gamma r x},
$$

where $M_{i}$ and $M_{j}$ are the solutions of the system of algebraic equations

$$
\left(\rho+\lambda_{i}-r+\frac{\theta_{i}^{2}}{2}\right) M_{i}+r M_{i} \log M_{i}-\lambda_{i} M_{j}=0,
$$


where $i, j \in\{1,2\}$ and $i \neq j$, and the optimal policies $\left(c_{i}^{*}, \pi_{i}^{*}\right)$ are given by

$$
c_{i}^{*}=r x-\frac{1}{\gamma} \log M_{i} \quad \text { and } \quad \pi_{i}^{*}=\frac{\theta_{i}}{\gamma r \sigma_{i}}, i=1,2 .
$$

Proof. From the optimization problem (3.1), we have the following coupled Bellman equations

$$
\begin{gathered}
\max _{\left(c_{i}, \pi_{i}\right)}\left[\left\{r x+\pi_{i}\left(\mu_{i}-r\right)-c_{i}\right\} V_{i}^{\prime}(x)+\frac{1}{2} \sigma_{i}^{2} \pi_{i}^{2} V_{i}^{\prime \prime}(x)\right. \\
\left.-\left(\rho+\lambda_{i}\right) V_{i}(x)+\lambda_{i} V_{j}(x)-\frac{e^{-\gamma c_{i}}}{\gamma}\right]=0,
\end{gathered}
$$

where $i, j \in\{1,2\}$ and $i \neq j$. The first-order conditions (FOCs) imply

$$
c_{i}^{*}=-\frac{1}{\gamma} \log \left(V_{i}^{\prime}(x)\right) \quad \text { and } \quad \pi_{i}^{*}=-\frac{\theta_{i} V_{i}^{\prime}(x)}{\sigma_{i} V_{i}^{\prime \prime}(x)}, i=1,2 .
$$

We assume that the optimal consumption $c_{i}^{*}=C_{i}(x), i=1,2$, is a function of wealth. And let $X_{i}(\cdot), i=1,2$, be the inverse function of $C_{i}(\cdot), i=1,2$. That is, $X_{i}(\cdot)=C_{i}^{-1}(\cdot), i=1,2$. Then the FOCs (3.6) imply

$$
V_{i}^{\prime}(x)=e^{-\gamma C_{i}(x)} \quad \text { and } \quad V_{i}^{\prime \prime}(x)=-\frac{\gamma}{X_{i}^{\prime}\left(c_{i}\right)} e^{-\gamma C_{i}(x)}, i=1,2 .
$$

Plugging the FOCs (3.6) with (3.7) into the Bellman equation (3.5), then we obtain

$$
\begin{aligned}
& r X_{i}\left(c_{i}\right) e^{-\gamma c_{i}}+\frac{\theta_{i}^{2}}{2 \gamma} X_{i}^{\prime}\left(c_{i}\right) e^{-\gamma c_{i}}-c_{i} e^{-\gamma c_{i}} \\
& -\left(\rho+\lambda_{i}\right) V_{i}\left(X_{i}\left(c_{i}\right)\right)+\lambda_{i} V_{j}\left(X_{i}\left(c_{i}\right)\right)-\frac{e^{-\gamma c_{i}}}{\gamma}=0,
\end{aligned}
$$

where $i, j \in\{1,2\}$ and $i \neq j$. Taking derivative of the equation (3.8) with respect to $c_{i}$, then we obtain

$$
\begin{aligned}
r X_{i}^{\prime}\left(c_{i}\right) e^{-\gamma c_{i}} & -\gamma r X_{i}\left(c_{i}\right) e^{-\gamma c_{i}}+\frac{\theta_{i}^{2}}{2 \gamma} X_{i}^{\prime \prime}\left(c_{i}\right) e^{-\gamma c_{i}}-\frac{\theta_{i}^{2}}{2} X_{i}^{\prime}\left(c_{i}\right) e^{-\gamma c_{i}} \\
& +\gamma c_{i} e^{-\gamma c_{i}}-\left(\rho+\lambda_{i}\right) X_{i}^{\prime}\left(c_{i}\right) e^{-\gamma c_{i}}+\lambda_{i} X_{i}^{\prime}\left(c_{i}\right) e^{-\gamma c_{j}}=0
\end{aligned}
$$

where $i, j \in\{1,2\}$ and $i \neq j$, since $x=X_{1}\left(c_{1}\right)=X_{2}\left(c_{2}\right)$ implies $V_{j}^{\prime}\left(X_{i}\left(c_{i}\right)\right)=V_{j}^{\prime}(x)=e^{-\gamma c_{j}}$, where $i, j \in\{1,2\}$ and $i \neq j$. Thus we 
obtain the coupled second order differential equations with respect to $c_{1}$ and $c_{2}$

$$
\begin{aligned}
& \frac{\theta_{i}^{2}}{2 \gamma} X_{i}^{\prime \prime}\left(c_{i}\right)-\left(\rho+\lambda_{i}-r+\frac{\theta_{i}^{2}}{2}\right) X_{i}^{\prime}\left(c_{i}\right) \\
& -\gamma r X_{i}\left(c_{i}\right)+\gamma c_{i}+\lambda_{i} e^{-\gamma\left(c_{j}-c_{i}\right)} X_{i}^{\prime}\left(c_{i}\right)=0,
\end{aligned}
$$

where $i, j \in\{1,2\}$ and $i \neq j$. If we conjecture the solution $X_{i}\left(c_{i}\right)$ of the form

$$
X_{i}\left(c_{i}\right)=\frac{c_{i}}{r}+\frac{1}{\gamma r} \log M_{i} \quad \text { and } \quad c_{i}=r x-\frac{1}{\gamma} \log M_{i}, i=1,2,
$$

for some constant $M_{i}>0$, then $X_{i}^{\prime}\left(c_{i}\right)=1 / r$ and $X_{i}^{\prime \prime}\left(c_{i}\right)=0, i=1,2$. The equation (3.10) implies

$$
c_{j}-c_{i}=\frac{1}{\gamma} \log \frac{M_{i}}{M_{j}},
$$

where $i, j \in\{1,2\}$ and $i \neq j$. So the equation (3.9) can be reduced into the system of algebraic equations (3.3).

Now we want to show that there exists a unique pair solution $\left(M_{1}, M_{2}\right)$ to (3.3). Without loss of generality, we may assume that $\theta_{i}<\theta_{j}$. Let

$$
M_{j}=f\left(M_{i}\right):=\frac{1}{\lambda_{i}}\left(\rho+\lambda_{i}-r+\frac{\theta_{i}^{2}}{2}\right) M_{i}+\frac{r}{\lambda_{i}} M_{i} \log M_{i}>0,
$$

for $M_{i}>0$, and let

$$
f_{1}\left(M_{i}\right):=\frac{1}{\lambda_{i}}\left(\rho+\lambda_{i}-r+\frac{\theta_{i}^{2}}{2}\right)+\frac{r}{\lambda_{i}} \log M_{i}>0,
$$

then $f_{1}^{\prime}\left(M_{i}\right)=r /\left(\lambda_{i} M_{i}\right)>0$. Now we consider the constants $\bar{x}$ and $\underline{x}$ with $\bar{x}>\underline{x}$ as follows:

$$
\bar{x}:=e^{-\frac{1}{r}\left(\rho-r+\frac{\theta_{i}^{2}}{2}\right)}<1 \quad \text { and } \quad \underline{x}:=e^{-\frac{1}{r}\left(\rho+\lambda_{i}-r+\frac{\theta_{i}^{2}}{2}\right)}<1,
$$

where the inequalities are obtained from Assumption 3.1. Then $f_{1}(\bar{x})=$ $1, f_{1}(\underline{x})=0$. Since $f_{1}\left(M_{i}\right)>0, M_{i}>\underline{x}$.

Now let

$g\left(M_{i}\right):=\left(\rho+\lambda_{j}-r+\frac{\theta_{j}^{2}}{2}\right) M_{i} f_{1}\left(M_{i}\right)+r M_{i} f_{1}\left(M_{i}\right) \log M_{i} f_{1}\left(M_{i}\right)-\lambda_{j} M_{i}$,

and let

$$
g_{1}\left(M_{i}\right):=\left(\rho+\lambda_{j}-r+\frac{\theta_{j}^{2}}{2}\right) f_{1}\left(M_{i}\right)+r f_{1}\left(M_{i}\right) \log M_{i} f_{1}\left(M_{i}\right)-\lambda_{j} .
$$


Note that $g_{1}(\bar{x})=\left(\theta_{j}^{2}-\theta_{i}^{2}\right) / 2>0$. Since, by l'Hospital's rule,

$$
\lim _{M_{i} \rightarrow \underline{x}+} \frac{\log M_{i} f_{1}\left(M_{i}\right)}{1 / f_{1}\left(M_{i}\right)}=\lim _{M_{i} \rightarrow \underline{x}+} \frac{f_{1}\left(M_{i}\right)\left(f_{1}\left(M_{i}\right)+M_{i} f_{1}^{\prime}\left(M_{i}\right)\right)}{-M_{i} f_{1}^{\prime}\left(M_{i}\right)}=0,
$$

$\lim _{M_{i} \rightarrow \underline{x}+} g_{1}\left(M_{i}\right)=-\lambda_{j}<0$. Thus, by intermediate value theorem, there exists $\bar{M}>0$ such that $g_{1}(\bar{M})=0$ and $\underline{x}<\bar{M}<\bar{x}$. Taking derivative of $g_{1}\left(M_{i}\right)$, then

$$
\begin{aligned}
& g_{1}^{\prime}\left(M_{i}\right) \\
& =\left(\rho+\lambda_{j}+\frac{\theta_{j}^{2}}{2}\right) f_{1}^{\prime}\left(M_{i}\right)+r f_{1}^{\prime}\left(M_{i}\right) \log M_{i} f_{1}\left(M_{i}\right)+r \frac{f_{1}\left(M_{i}\right)}{M_{i}} \\
& =\frac{r}{\lambda_{i} M_{i}} h\left(M_{i}\right),
\end{aligned}
$$

where

$$
h\left(M_{i}\right):=\left(2 \rho-r+\lambda_{i}+\lambda_{j}+\frac{\theta_{i}^{2}+\theta_{j}^{2}}{2}\right)+r \log M_{i}^{2} f_{1}\left(M_{i}\right) .
$$

Taking derivative of $h\left(M_{i}\right)$, then

$$
h^{\prime}\left(M_{i}\right)=r\left(\frac{2}{M_{i}}+\frac{f_{1}^{\prime}\left(M_{i}\right)}{f_{1}\left(M_{i}\right)}\right)>0 .
$$

Thus $h\left(M_{i}\right)$ is increasing, $\lim _{M_{i} \rightarrow \underline{x}+} h\left(M_{i}\right)=-\infty$ and $h(\bar{x})=r+\lambda_{i}+$ $\lambda_{j}+\left(\theta_{j}^{2}-\theta_{i}^{2}\right) / 2>0$. Again, by intermediate value theorem, there exists a unique $x^{*}>0$ such that $h\left(x^{*}\right)=0$ and $\underline{x}<x^{*}<\bar{x}$. That is, $h\left(M_{1}\right)<0$ for $\left(\underline{x}, x^{*}\right)$ and $h\left(M_{1}\right)>0$ for $\left(x^{*}, \infty\right)$. This implies $g_{1}^{\prime}\left(M_{1}\right)<0$ for $\left(\underline{x}, x^{*}\right)$ and $g_{1}^{\prime}\left(M_{1}\right)>0$ for $\left(x^{*}, \infty\right)$. Thus $g_{1}\left(M_{1}\right)$ is decreasing and negative for $\left(\underline{x}, x^{*}\right)$ and $g_{1}\left(M_{1}\right)$ is increasing for $\left(x^{*}, \infty\right)$. Therefore $\bar{M}$ with $x^{*}<\bar{M}<\bar{x}$ is the unique solution to $g_{1}\left(M_{i}\right)=0$, and consequently we obtain the unique pair solution $\left(M_{1}, M_{2}\right)$ to $(3.3)$.

Plugging $c_{i}$ in (3.10) into (3.7), then we derive

$$
V_{i}^{\prime}(x)=M_{i} e^{-\gamma r x} \quad \text { and } \quad V_{i}^{\prime \prime}(x)=-\gamma r M_{i} e^{-\gamma r x} .
$$

Also plugging (3.12) into the FOCs (3.6), then we obtain the optimal policies $\left(c_{i}^{*}, \pi_{i}^{*}\right)$ in (3.4). Therefore, from the Bellman equations (3.5), we obtain the value function $V_{i}(x)$ in (3.2).

\section{References}

[1] B. G. Jang, H. K. Koo, H. Liu, M. Loewenstein, Liquidity Premia and Transaction Costs, J. Finance 62 (2007), 2329-2366. 
[2] I. Karatzas, J. P. Lehoczky, S. P. Sethi, S. E. Shreve, Explicit Solution of a General Consumption/Investment Problem, Math. Oper. Res. 11 (1986), 261294.

[3] R. C. Merton, Lifetime Portfolio Selection under Uncertainty: the ContinuousTime Case, Rev. Econ. Stat. 51 (1969), 247-257.

[4] R. C. Merton, Optimum Consumption and Portfolio Rules in a ContinuousTime Model, J. Econ. Theory 3 (1971), 373-413.

[5] Y. H. Shin, Portfolio Selection with Regime-Switching: Dynamic Programming Approaches, J. Chungcheong Math. Soc. 25 (2012), 277-281.

[6] L. R. Sotomayor, A. Cadenillas, Explicit Solutions of Consumption-Investment Problems in Financial Markets with Regime Switching, Math. Finance 19 (2009), 251-279.

Department of Mathematics Sookmyung Women's University Seoul 140-742, Republic of Korea E-mail: yhshin@sookmyung.ac.kr 